\title{
GRAM POSITIVE BACTERIA CAUSING LOWER RESPIRATORY TRACT INFECTIONS AND THEIR RESISTANGE PATTERNS IN KATHMANDU UNIVERSITY HOSPITAL
}

\author{
Sherchan JB, ${ }^{1}$ Humagain $S^{2}$
}

${ }^{1}$ Department of Microbiology, Kathmandu University School of Medical Sciences, ${ }^{2}$ Department of Internal Medicine, Dhulikhel Hospital, Dhulikhel, Nepal.

\begin{abstract}
Respiratory tract infection due to Gram positive bacteria is a common cause of morbidity and mortality worldwide. This study of one year duration was carried out to determine the antibiotic resistance pattern of Gram-positive bacteria from patients with lower respiratory tract infection visiting Kathmandu University Hospital. A total of 1,556 respiratory samples including sputum and endotracheal aspirates were processed following standard guidelines. Antibiotic resistance pattern was determined following Clinical Laboratory Standard Institute guidelines. Gram positive bacteria from total sample were $6.6 \%$. Out of them $86.4 \%$ were Streptococcus pneumoniae and $13.6 \%$ were Staphylococcus aureus. Majority of S. pneumoniae, 53.9\% were resistant to azithromycin, followed by cotrimoxazole $40.4 \%$, ciprofloxacin $13.5 \%$ and Ceftriaxone $1.1 \%$ but all isolates were sensitive to penicillin. A total of $92.9 \%$ S aureus were methicillin and Ciprofloxacin resistant, $64.3 \%$ were resistant to cotrimoxazole and $28.6 \%$ resistant to amikacin but all strains were sensitive to linezolid. Majority of multidrug resistant Staphylococcus were detected among patients of age more than 60 years. A total of $77.8 \%$ patients had lower respiratory tract infection with acute exacerbation of chronic obstructive pulmonary disease. Majority of diabetic patients had multidrug resistant organism. $70.69 \%$ patients of age above 60 had multidrug resistant isolates. Multidrug-resistant Gram positive bacteria were observed in respiratory samples. For effective management of lower respiratory tract infections detailed microbiological diagnosis and susceptibility testing is required.
\end{abstract}

\section{KEYWORDS}

Methicillin resistant Staphylococcus aureus; multidrug-resistance

\section{CORRESPONDING AUTHOR}

Dr. Jatan Bahadur Sherchan,

Associate Professor

Department of Microbiology

Kathmandu University School of Medical Sciences

Dhulikhel, Nepal

Email: jatansherchan@gmail.com

Orcid ID: 0000-0001-8576-1631

DOI: https://doi.org/10.3126/nmcj.v22i1-2.30028 


\section{INTRODUCTION}

Acute lower respiratory tract infection is defined as evidence of acute infection (reported fever, documented temperature $>38.0^{\circ} \mathrm{C}$, elevated age-specific white blood cell count or abnormal differential) and symptoms/signs of respiratory illness (abnormal breath sounds, tachypnea based on documented respiratory rate, cough, sputum production, hemoptysis, chest pain or dyspnea) at the time of admission. Previous reports from those surveillance sites have used this case definition..$^{1-4}$

Treatment of patients from whom multidrug resistant bacteria has been isolated is becoming a major challenge to the physicians these days. Among Gram positive bacterial strains, Methicillin resistant Staphylococcus aureus (MRSA) producing bacterial strains and drug resistant $S$. pneumoniae are common ones. ${ }^{5}$ These bacterial isolates causing LRTI is associated with risk factors and has modified therapeutic approach. Risk factors such immunocompromised status may be a major factor which is responsible for some of the cases of multidrug resistant (MDR) bacterial respiratory infections. Antibiotic is the mainstay of therapy for bacterial infections but they have been misused and overused. ${ }^{5}$ Hence, bacteria gained resistance to the antibiotics. Therefore, the treatment of risk factors and avoiding misuse of antibiotics are important for infection control. Almost three quarters of all antibiotic consumptions are for respiratory tract infections. ${ }^{5}$

This study was conducted with the aim of studying the Gram positive etiological agents causing LRTI in patients of all age groups and their antibiotic susceptibility pattern with risk factors in Kathmandu University Hospital.

\section{MATERIALS AND METHODS}

The study was cross-sectional study which was conducted at Kathmandu University Hospital, Dhulikhel from the month of October 2018 to September 2019. Ethical clearance was taken from Institutional Review Committee of Kathmandu University Hospital before the study was conducted. After obtaining informed consent clinical data collection was done by history taking and clinical examination by the researcher himself with the help of consultant doctor taking care of the patient as well as by going through the medical and lab records.

Respiratory samples such as expectorated sputum, endotracheal aspirates and secretions, bronchoalveolar washings or BAL samples were collected and processed for culture and sensitivity as recommended by American Society for Microbiology. ${ }^{6}$ Prior to sputum collection patient was instructed not to expectorate saliva or postnasal discharge into the container and sputum was collected from deep cough in a sterile leakproof container. ${ }^{6}$ Endotracheal aspirate and BAL samples were collected in a sterile container. ${ }^{6}$ The specimen were labelled with demographic information, date and time of collection and site of collection and transported to microbiology lab within half an hour to prevent loss of recovery of fastidious pathogens, such as $S$. pneumoniae and overgrowth of oronasal microbiota. ${ }^{6}$ Processing was done by inoculating the specimen on $5 \%$ Sheep Blood agar (BA) with optochin disc and MacConkey agar (MA) plates. The BA plates were incubated in $\mathrm{CO}_{2}$ incubator $\left(10 \% \mathrm{CO}_{2}\right)$ at $37^{\circ} \mathrm{C}$ for 48-72 hours while MA plates were incubated at $37^{\circ} \mathrm{C}$ for $24-48$ hours in aerobic atmosphere. ${ }^{6}$ Identification of the bacteria was done by Gram staining smears made from culture plates and for identification of $S$. pneumoniae, alphahemolytic colonies consistent with S. pneumoniae was examined along with optochin sensitivity and solubility in $10 \%$ bile following standard procedures. ${ }^{6}$

For identification of $S$. aureus, all isolates were observed for hemolysis after overnight incubation at $37^{\circ} \mathrm{C}$ on sheep blood agar along with Gram staining of smears made from culture plate with coagulase test using rabbit plasma and DNase activity similar to the control strain ATCC 29213. All the respiratory isolates were tested for antibiotic susceptibility by modified Kirby Bauer disc diffusion method in compliance with CLSI 2018 guidelines on Mueller Hinton agar plates. ${ }^{7}$

Detection of MRSA was done by cefoxitin disc diffusion test in which direct colony suspension in saline was prepared and matched with the turbidity standard equivalent to 0.5 MacFarland standard. A plate of Mueller Hinton Agar was inoculated and cefoxitin disc $30 \mu \mathrm{g}$ (HIMEDIA) was applied to the plate. The plate was incubated at $37^{\circ} \mathrm{C}$ for 24 hours. The results were interpreted according to CLSI guidelines for cefoxitin susceptibility testing: a zone size $\leq 21 \mathrm{~mm}$ was considered resistant and $\geq 22 \mathrm{~mm}$ was considered susceptible. $^{7}$

Data were analyzed by (SPSS) version 11.5 software and $p$ value less than 0.05 was considered significant.

\section{RESULTS}

Total number of sputum and other samples (endotracheal aspirate, BAL etc.) received during the study was 1,556. Out of this growth of Gram positive bacteria was only $103(6.62 \%)$. Out of this total number of sputum sample with Gram positive isolates were 99 (96.12\%), from endotracheal 
tube secretion Gram positive isolates were 3 (2.91\%) and from tracheal aspirate, Gram positive isolates was only $1(0.97 \%)$. Sixty-three (61.17\%) patients from whom Gram positive bacteria was isolate were male and forty (38.83\%) were female patients.

Eighty-nine (86.40\%) of the isolates were $S$. pneumoniae and fourteen (13.60\%) of the isolates were $S$. aureus. Patients age profile showed that 2 (1.94\%) were neonates and infants, 2 (1.94\%) were from 16 to 30 years of age, 41 (39.81\%) were from $31-60$ years of age and 58 (56.31\%) were above age 60 years.

Antibiotic resistance pattern of $S$. pneumoniae showed that majority of the strains, 48 (53.93\%) were resistant to azithromycin, followed by cotrimoxazole 36 (40.45\%), ciprofloxacin 12 (13.48\%) and ceftriaxone $1(1.12 \%)$ respectively. All isolates were sensitive to penicillin as shown in Table 1.

Table 1: Antibiotic Resistance pattern (\% resistance) for Streptococcus pneumoniae $(n=89)$

\begin{tabular}{|lc|} 
Antibiotics & $\begin{array}{c}\text { Number and percentage } \\
\text { resistant }\end{array}$ \\
Penicillin & $0(0.0 \%)$ \\
Amoxycillin & $0(0.0 \%)$ \\
Ciprofloxacin & $12(13.5 \%)$ \\
Cotrimoxazole & $36(40.4 \%)$ \\
Ceftriaxone & $1(1.1 \%)$ \\
Azithromycin & $48(53.9 \%)$ \\
\hline
\end{tabular}

Antibiotic resistance pattern of $S$. aureus showed that 13 (92.86\%) were methicillin resistant (MRSA) and ciprofloxacin resistant, 9 (64.28\%) were resistant to cotrimoxazole and 4 (28.57\%) were resistant to amikacin. All strains were sensitive to erythromycin and linezolid.

Table 2: Antibiotic Resistance pattern (\% resistance) for Staphylococcus aureus $(n=19)$

\begin{tabular}{|lc|}
\hline & $\begin{array}{c}\text { Staphylococcus } \\
\text { aureus }(\mathbf{n}=14)\end{array}$ \\
Amoxycillin & $13(92.9 \%)$ \\
Cefoxitin & $13(92.9 \%)$ \\
Amikrofloxacin & $13(92.9 \%)$ \\
Cotrimoxazole & $4(28.6 \%)$ \\
Erythromycin & $9(64.3 \%)$ \\
Linezolid & $0(0.0 \%)$ \\
\hline
\end{tabular}

Out of total 13 MRSA, 9 (69.23\%) were detected among patients of age more than 60 years. Regarding infection, $84(81.55 \%)$ patients had lower respiratory tract infection along with acute exacerbation of chronic obstructive pulmonary disease. A total of 24 (23.30\%) patients had only lower respiratory tract infection with no acute exacerbation of chronic obstructive pulmonary disease.

Out of 26 diabetic patients, 18 (69.23\%) had multidrug resistant organism and out of 77 nondiabetic patients only 26 (33.77\%) had multidrug resistant organism. When statistical analysis was done using the above groups and outcome, the $\mathrm{p}$ value was equal to 0.0025 which is considered to be statistically very significant.

Out of 58 patients with age above 60 years, 41 (70.69\%) patients had multidrug resistant isolates and out of 45 patients with age below 60 years, $12(26.67 \%)$ patients had multidrug resistance. When statistical analysis was done using the above groups and outcome, the p-value was less than 0.0001 which is considered to be statistically extremely significant.

\section{DISCUSSION}

This study revealed Gram positive bacterial isolates from lower respiratory tract infection and determined antibiotic resistance pattern and multidrug resistance. It also detected some of the risk factors among patients which might have made the patient susceptible to such infection. Total number of sputum and other lower respiratory samples received during the one year study duration was 1,556 but the growth of Gram positive bacteria was only $6.62 \%$ which seem quite less as our study included only Gram positive bacteria and not Gram negative bacteria.

A total of $96.12 \%$ of isolates were from sputum, $2.91 \%$ from endotracheal tube secretion and $0.97 \%$ from tracheal aspirate. Sputum was the most common sample received for diagnosis of lower respiratory tract infection in microbiology lab although sputum culture may not be helpful in distinguishing upper airway colonization from lower airway infection. ${ }^{8}$

A total of $61.17 \%$ patients were males and $38.83 \%$ were females which seem similar to the study conducted by Biswas et $a l^{9}$ (2013) in which $59.12 \%$ were males and $40.88 \%$ were females. It is obviously well known that males are more involved in outdoor activities and is more prone to getting contagious respiratory tract infection from the community. 
In this study $S$. pneumoniae was the most common bacteria accounting $86.40 \%$ of the isolates. In western part of Nepal a study was conducted by Khan S et $a l^{10}$ (2014), in which S. pneumoniae was the most predominant Gram positive bacteria causing lower respiratory tract infection. Our study is consistent with the well-known fact that S. pneumoniae is the leading cause of pneumonia globally and mostly in Asia and Africa. ${ }^{11}$

In this study majority of the patients belonged to age above 60 years which might indicate that the old age might be a predisposing factor for acquiring lower respiratory tract infection particularly community acquired pneumonia, which seems similar to the finding by Lamas et al (2017). ${ }^{12}$

This study observed that majority of S. pneumoniae, $53.93 \%$ were resistant to azithromycin which is a macrolide group of antibiotic. Problem of macrolide resistant pneumococcus has also been detected in study conducted by Niederman (2015). ${ }^{13}$ Although the resistance pattern of cotrimoxazole, ceftriaxone and penicillin did not match with other studies the resistance to ciprofloxacin $13.48 \%$ was quite close to the study conducted by Zahraa et al ${ }^{14}$ (2017) in which $16.2 \%$ of the strains of $S$. pneumoniae were resistant to ciprofloxacin.

MRSA in Nepal was first reported by Rai et $a l^{15}$ (1990). This study found that most of the $S$. aureus, $92.86 \%$ causing lower respiratory tract infections were methicillin resistant (MRSA) and ciprofloxacin resistant. The frequency of lower respiratory tract infection caused by MRSA is increasing which may be both community acquired and hospital acquired. ${ }^{16}$

But only, $64.28 \%$ were resistant to cotrimoxazole and $28.57 \%$ were resistant to amikacin. All strains were sensitive to erythromycin and linezolid which was similar to the finding of Song et $a l^{17}$ (2017). But, since our study has less number of samples and short duration, we cannot recommend linezolid as appropriate drug for the treatment of MRSA.

In our study, 69.23\% MRSA were detected among patients of age more than 60 years. This indicated that old aged people and neonates were more prone to colonization and infection by drug resistant Staphylococcus. A study was conducted by Simor et al $^{18}$ (2005) on MRSA using much larger number of patients and wider variety of diseases compared to our study also showed that $66 \%$ of MRSA was detected among patients of age more than 65 years. In our study, $77.78 \%$ patients had lower respiratory tract infection along with acute exacerbation of chronic obstructive pulmonary disease and $22.22 \%$ patients had only lower respiratory tract infection, indicating that acute exacerbation of chronic obstructive pulmonary disease might be a predisposing factor for lower respiratory tract infection and might be the most frequent comorbid condition that is present in patients with pneumonia similar to the finding of Marcos et al (2018). ${ }^{19}$

Antibiotic resistance among diabetic patients is a major concern. In our study although the sample size and duration of study was not significant, multidrug resistance was detected more among diabetic patients $(69.23 \%)$ in comparison to non-diabetic patients $(31.71 \%)$. When statistical analysis was done using the above groups and outcome, the $\mathrm{p}$ value was equal to 0.0011 which seems much more significant than the finding of $\mathrm{Yu}$-Xin et $a l^{20}$ (2019).

In our study, 70.69. \% patients with age above 60 years had multidrug resistant bacterial isolates and only $24 \%$ of patients with age below 60 years had multidrug resistant bacterial isolates. Although the number of patients was too less, we did statistical analysis using the above groups and outcome and we found p-value to be less than 0.0001. Infections caused by multi-resistant bacteria are increasing among the elderly patients as mentioned in a study conducted by Augustine et al (2011). ${ }^{21}$ Multi-drug resistant gram positive bacteria were not detected among malignancy patients in this study.

Some of the limitations of this study might be the study duration was short and patients were much less to determine all possible bacteria causing lower respiratory tract infection. Beside this only Gram positive aerobic and facultative anaerobic bacteria were investigated and no Gram negative and anaerobic bacteria or fungal pathogens were determined. Vancomycin susceptibility was not reported as we did not determine MIC (Minimum inhibitory concentration) of it. All possible risk factors were not determined and we cannot totally rely on the above finding for multidrug resistance.

In Conclusion, prevention of lower respiratory tract infection by multidrug-resistant Gram positive bacteria especially in elderly people seems to be a very important issue as shown by the study. This might be achieved by treatment of risk factors, avoiding misuse of antibiotic especially prior antibiotic therapy and early investigation and analysis of infection.

\section{ACKNOWLEDGEMENTS}

We express our profound gratitude to Department of Microbiology and Department of Internal Medicine of Kathmandu University Hospital for their kind support and co-operation throughout this study. 


\section{REFERENCES}

1. Fry AM, Chittaganpitch M, Baggett HC, et al. The burden of hospitalized lower respiratory tract infection due to respiratory syncytial virus in rural Thailand. PLoS One 2010; 5:e15098.

2. Fry $\mathrm{AM}, \mathrm{Lu} \mathrm{X}$, Olsen SJ, et al. Human rhinovirus infections in rural Thailand: epidemiological evidence for rhinovirus as both pathogen and bystander. PLoS One 2011; 6:e17780.

3. Baggett HC, Peruski LF, Olsen SJ, et al. Incidence of pneumococcal bacteremia requiring hospitalization in rural Thailand. Clin Infect Dis 2009; 48(suppl 2):S65-S74.

4. Olsen SJ, Thamthitiwat S, Chantra S, et al. Incidence of respiratory pathogens in persons hospitalized with pneumonia in two provinces in Thailand. Epidemiol Infect 2010; 138: 1811-22.

5. File TM. The epidemiology of respiratory tract infection. Semin Respir Infect 2000; 15: 184-94.

6. Isenberg HD. Clinical Microbiology Procedures Handbook. 2nd ed. Washington, D.C: ASM Press 2004.

7. CLSI. Performance Standards for Antimicrobial Susceptibility Testing. 28th ed. CLSI supplement M100. Wayne, PA: Clinical and Laboratory Standards Institute; 2018.

8. Reimer LG, Carroll KC. Role of the microbiology laboratory in the diagnosis of lower respiratory tract infections. Clin Infect Dis 1998; 26: 742-8.

9. Biswas PP, K TP. Bacterial causes of lower respiratory tract infections in patients attending central referral hospital, Gangtok with reference to antibiotic resistance pattern.J Evol Med Dental Sci 2013; 2: 8126-35.

10. Khan S, Priti S, Ankit S. Bacteria Etiological Agents Causing Lower Respiratory Tract Infections and Their Resistance Patterns. Iran Biomed J 2015; 19: 240-6.

11. GBD 2016 Lower Respiratory Infections Collaborators. Estimates of the global, regional, and national morbidity, mortality, and aetiologies of lower respiratory infections in 195 countries, 1990-2016: A systematic analysis for the Global Burden of Disease Study 2016. Lancet Infect Dis 2018; 18: 1191-210.
12. Lamas CC, Coelho LE, Grinsztejn BJ et al. Community-acquired lower respiratory tract infections in HIV infected patients on antiretroviral therapy: predictors in a contemporary cohort study. Infection 2017; 45: 801-9.

13. Niederman M.S.Macrolide-ResistantPneumococcus in Community-acquired Pneumonia. Is There Still a Role for Macrolide Therapy? Am J Respir Crit Care Med 2015; 191: 1216-7.

14. Motaweq ZY, Naher HS. Antimicrobial susceptibility of Streptococcus pneumoniae isolates causing LRTI in Najaf, Iraq. Environ Socio-economic Studies 2017; 5: 10-8.

15. Rai SK, Tuladhar NR, Shrestha HG. Methicillin resistant Staphylococcus aureus in a tertiary care center, Nepal. Indian J Med Microbiol 1990; 8: 108-9.

16. Rubinstein E, Kollef MH, Nathwani D. Pneumonia Caused by Methicillin-Resistant Staphylococcus aureus. Clin Infect Dis 2008; 46: 378-85.

17. Song Z, Gu FF, Guo XKet al. Antimicrobial Resistance and Molecular Characterization of Staphylococcus aureus Causing Childhood Pneumonia in Shanghai. Front Microbiol 2017; 8: 1-7.

18. Simor A, Ofner-Agostini M, Paton S et al. Clinical and Epidemiologic Features of Methicillin-Resistant Staphylococcus aureus in Elderly Hospitalized Patients. Infect Control Hospital Epidemiol 2005; 26: 838-41.

19. Restrepo MI, Sibila O, Anzueto A. Pneumonia in Patients with Chronic Obstructive Pulmonary Disease. Tuberc Respir Dis (Seoul) 2018; 81: 187-97.

20. Liu YX, Cao QM, Ma BC. Pathogens distribution and drug resistance in patients with acute cerebral infarction complicated with diabetes and nosocomial pulmonary infection. BMC Infect Dis 2019; 19: 603.

21. Augustine S, Bonomo RA. Taking stock of infections and antibiotic resistance in the elderly and long term care facilities: a survey of existing and upcoming challenges. Eur J Micro Immu 2011; 1: 190-7. 